\title{
HAMILTON-JACOBI METHOD FOR CLASSICAL MECHANICS IN GRASSMANN ALGEBRA
}

\author{
K. V. Tabunshchyk \\ Institute for Condensed Matter Physics of the Ukrainian Academy of Sciences, \\ 1 Svientsitskii Str., Lviv, UA-79011, Ukraine \\ (Received September 30, 1999)
}

\begin{abstract}
We present the Hamilton-Jacobi method for the classical mechanics with the constrains in Grassmann algebra. Within the framework of this method the solution for the classical system characterized by the SUSY Lagrangian is obtained.

Key words: Hamilton-Jacobi method, SUSY system, classical mechanics, constrains.
\end{abstract}

PACS number(s): 45.20.Jj, 12.60.Jv, 11.30.Pb

The problem of Lagrangian and Hamiltonian mechanics with Grassmann variables has been discussed previously in works [1-3] where examples of solutions for classical systems were presented.

In this paper we propose the Hamilton-Jacobi method for the solution of the classical counterpart of Witten's model [4].

We assume that the states of mechanical system are described by the set of ordinary bosonic degrees of freedom $q$ (even Grassmann numbers) and the set of fermionic degrees of freedom $\psi$ (odd Grassmann numbers).

Within the framework of the classical mechanics with constrains the equations of motion can be obtained from the variational principle for the action

$$
S=\int\left[\dot{q}_{a} P_{q}^{a}-\tilde{H}\left(X, x, P_{x}\right)\right] \mathrm{dt}
$$

with the additional conditions (constrains)

$$
F_{\alpha}=F_{\alpha}\left(q, P_{q}\right)=0
$$

Here we introduce the Hamiltonian

$$
\begin{aligned}
& \tilde{H}\left(X, x, P_{x}\right)=\left(\frac{\partial L}{\partial \dot{q}^{a}} \dot{q}^{a}-L\right)_{\dot{x}=\tilde{\dot{x}}}, \\
& q=\{X, x\}, \quad x^{i}=q^{i}, \quad i={\overline{1, R_{\text {max }}}}, \\
& \operatorname{det}\left\|\frac{\partial^{2} L}{\partial \dot{x} \partial \dot{x}}\right\| \neq 0, \quad \operatorname{rank}\left\|\frac{\partial^{2} L}{\partial \dot{q} \partial \dot{q}}\right\|=R_{\text {max }}
\end{aligned}
$$

and $P_{x}$ is the canonical momentum conjugate to the variables $x$. Velocities $\tilde{\dot{x}}\left(q, P_{x}, \dot{X}\right)$ are the solutions of the equations $P_{x}=\partial L / \partial \dot{x}$ with respect to the variables $\dot{x}$. On the other hand equations $P_{X}=\partial L / \partial \dot{X}$ form the system of constrains (2).

The Hamilton-Jacobi equation in the case of the classical mechanics with constrains in Grassmann algebra is as follows:

$$
\frac{\partial S}{\partial t}=-H\left(q, \psi, \frac{\partial S}{\partial x}, \frac{\partial S}{\partial \psi}, \lambda_{a}\left(q, \psi, \frac{\partial S}{\partial x}, \frac{\partial S}{\partial \psi}\right), \lambda_{b}, t\right)
$$

Here $\left(\lambda_{a}, \lambda_{b}\right)$ is the set of certain Lagrange multipliers for the constrains from the variational problem (1) in Grassmann algebra. Lagrange multipliers $\lambda_{a}$ can be found from the equations of motion

$$
\begin{gathered}
\dot{\eta}=\left\{\eta, \tilde{H}+\lambda^{\alpha} F_{\alpha}\right\}, \quad F_{\alpha}\left(q, \psi, P_{q}, P_{\psi}\right)=0 \\
\eta=\left(q, \psi, P_{q}, P_{\psi}\right)
\end{gathered}
$$

and from the time-independence conditions

$$
\dot{F}_{\alpha}\left(q, \psi, P_{q}, P_{\psi}\right)=0
$$

On the other hand, $\lambda_{b}$ are those multipliers which cannot be found and which form a functional arbitration for solutions (in the theory with the first-class constrains [5-7]). However, we can transform the theory with the first-class constrains to the physically equivalent theory with the second-class constrains. As an example, we can take the strong minimal gauge which does not shift the equations of motion (the so-called canonical gauge $G^{(c)}$ $[8]$ ).

The solution of Hamilton-Jacobi equation can be found with using of the Jacobi theorem. In our case this theorem has some differences from the standard formulation.

Let us consider a full solution of the Hamilton-Jacobi equation $S=S_{r}(q, \psi, \alpha, \beta, t)$ ( $\alpha$ is a set of the even Grassmann constants, $\beta$ is a set of the odd ones). We perform the canonical transformation from the old variables $q$, $\psi, P_{q}, P_{\psi}$ to the new ones (taking $S_{r}$ as a generating function) and put $\alpha=P_{Q}, \beta=P_{\nu}$ as new canonical momenta and $Q, \nu$ as new coordinates. Then the relations between the new and old variables can be written in the form:

$$
H^{\prime}=H+\frac{\partial S_{r}}{\partial t}, \quad P_{q}=\frac{\partial S_{r}}{\partial q}, \quad P_{\psi}=\frac{\partial S_{r}}{\partial \psi},
$$




$$
Q=\frac{\partial S_{r}}{\partial P_{Q}}, \quad \nu=-\frac{\partial S_{r}}{\partial P_{\nu}}
$$

Since $S_{r}$ is the solution of the Hamilton-Jacobi equation, we obtain that

$$
H^{\prime}=0 \Longrightarrow P_{Q}=\text { const, } Q=\text { const, } P_{\nu}=\text { const }, \nu=\text { const }
$$

new coordinates are constant. From the obtained result we can write:

$$
\begin{aligned}
& \partial S_{r} / \partial \alpha=\text { const (even Grassmann number) } \\
& \partial S_{r} / \partial \beta=\text { const (odd Grassmann number) }
\end{aligned}
$$

The solution of equations (4) gives the variables $q$ and $\psi$ as functions of time. Time dependencies of the canonical momenta can be found from the relations $P_{\psi}=\partial S / \partial \psi$, $P_{q}=\partial S / \partial q$.

Let us now consider the Lagrangian $[1,2]$

$$
L=\frac{\dot{q}^{2}}{2}-\frac{1}{2} V^{2}(q)-\frac{i}{2}(\dot{\bar{\psi}} \psi-\bar{\psi} \dot{\psi})-U(q) \bar{\psi} \psi
$$

which possess supersymmetry when $U(q)=V^{\prime}(q)$ (in this case the real function $V$ is the so-called superpotential) [4]. The overbar denotes the Grassmann variant of the complex conjugation.

The momenta conjugate to the fermionic variables do not depend on $\dot{\psi}$ or $\dot{\bar{\psi}}$. Hence, we have the following constrains between coordinates and momenta:

$$
F_{1}=P_{\psi}+\frac{i}{2} \bar{\psi}=0, \quad F_{2}=P_{\bar{\psi}}+\frac{i}{2} \psi=0
$$

The Lagrange multipliers can be found from the following time-independence conditions:

$$
\dot{F}_{1}=\left\{H(\lambda), F_{1}\right\}=0, \quad \dot{F}_{2}=\left\{H(\lambda), F_{2}\right\}=0,
$$

where

$$
\begin{aligned}
H(\lambda) & =\frac{P_{q}^{2}}{2}+\frac{1}{2} V^{2}(q)+U(q) \bar{\psi} \psi \\
& +\lambda_{1}\left(P_{\psi}+\frac{\mathrm{i}}{2} \bar{\psi}\right)+\lambda_{2}\left(P_{\bar{\psi}}+\frac{i}{2} \psi\right) .
\end{aligned}
$$

The solutions of (7) are the following ones:

$$
\lambda_{1}=-\mathrm{iU}(\mathrm{q}) \psi, \quad \lambda_{2}=\mathrm{iU}(\mathrm{q}) \bar{\psi}
$$

Since the Hamiltonian of system (5) is as follows:

$$
H=H(\lambda(q, \psi, \bar{\psi}))=\frac{P_{q}^{2}}{2}+\frac{1}{2} V^{2}(q)
$$

$$
-i U(q) P_{\bar{\psi}} \bar{\psi}+i U(q) P_{\psi} \psi
$$

Starting from the obtained Hamiltonian (9), the Hamilton-Jacobi equation (3) can be written in the form:

$$
\begin{aligned}
\frac{\partial S}{\partial t} & +\frac{1}{2}\left(\frac{\partial S}{\partial q}\right)^{2}+\frac{1}{2} V^{2}(q) \\
& -i U(q) \frac{\partial S}{\partial \bar{\psi}} \bar{\psi}+i U(q) \frac{\partial S}{\partial \psi} \psi=0
\end{aligned}
$$

Let us make an ansatz for the action

$$
\begin{aligned}
S(t, q, \psi, \bar{\psi}) & =S_{0}(t, q)+\psi \bar{\psi} S_{1}(t, q) \\
& +\psi S_{2}(t, q)+\bar{\psi} S_{3}(t, q)
\end{aligned}
$$

where $S_{0}$ and $S_{1}$ are even Grassmann functions and $S_{2}$ and $S_{3}$ are the odd ones. After the substitution of ansatz (11) into equation (10) and decomposition of this equation on Grassmann parities we obtain the following system of equations:

$$
\begin{aligned}
& \frac{\partial S_{0}}{\partial t}+\frac{1}{2}\left(\frac{\partial S_{0}}{\partial q}\right)^{2}+\frac{1}{2} V^{2}(q)=0 \\
& \frac{\partial S_{2}}{\partial t}+\frac{\partial S_{0}}{\partial q} \frac{\partial S_{2}}{\partial q}-i U(q) S_{2}=0 \\
& \frac{\partial S_{3}}{\partial t}+\frac{\partial S_{0}}{\partial q} \frac{\partial S_{3}}{\partial q}+i U(q) S_{3}=0 \\
& \frac{\partial S_{1}}{\partial t}+\frac{\partial S_{0}}{\partial q} \frac{\partial S_{1}}{\partial q}=0 \\
& \frac{\partial S_{2}}{\partial q} \frac{\partial S_{3}}{\partial q} \psi \bar{\psi}=0
\end{aligned}
$$

The first equation can be integrated by the variable decomposition method. Thus, we obtain

$$
S_{0}=\int \sqrt{2 E-V^{2}(q)} \mathrm{d} q-E t
$$

where $E$ is the constant of integration. Starting from expression (13), for the fourth equation we obtain the following:

$$
S_{1}=\int \frac{A \mathrm{~d} q}{\sqrt{2 E-V^{2}(q)}}-A t
$$

Here, $A$ and $E$ are real variables.

The solutions of the second and third equations can be written as 


$$
\begin{aligned}
& S_{2}=\phi_{1}\left(\int \frac{\mathrm{d} q}{\sqrt{2 E-V^{2}(q)}}-t\right) \exp \left(i \int \frac{U(q) \mathrm{d} q}{\sqrt{2 E-V^{2}(q)}}\right) \\
& S_{3}=\phi_{2}\left(\int \frac{\mathrm{d} q}{\sqrt{2 E-V^{2}(q)}}-t\right) \exp \left(-i \int \frac{U(q) \mathrm{d} q}{\sqrt{2 E-V^{2}(q)}}\right),
\end{aligned}
$$

where $\phi_{1}$ and $\phi_{2}$ are arbitrary odd Grassmann functions. In our case, it is sufficient to take $\phi_{1}=$ const, $\phi_{2}=$ const. The last equation from (12) leads to some condition on functions $\phi_{1}$ and $\phi_{2}$, which is satisfied when they are constant.

Thus, we can present the action in the following form:

$$
\begin{aligned}
S & =\int \sqrt{2 E-V^{2}(q)} \mathrm{d} q-E t+\int \frac{A \mathrm{~d} q}{\sqrt{2 E-V^{2}(q)}} \psi \bar{\psi}-A t \psi \bar{\psi} \\
& +\psi \phi_{1}\left(\int \frac{\mathrm{d} q}{\sqrt{2 E-V^{2}(q)}}-t\right) \exp \left(i \int \frac{U(q) \mathrm{d} q}{\sqrt{2 E-V^{2}(q)}}\right) \\
& +\bar{\psi} \phi_{2}\left(\int \frac{\mathrm{d} q}{\sqrt{2 E-V^{2}(q)}}-t\right) \exp \left(-i \int \frac{U(q) \mathrm{d} q}{\sqrt{2 E-V^{2}(q)}}\right) .
\end{aligned}
$$

Using the Jacobi theorem (4)

$$
\partial S / \partial \phi_{1}=\text { const }, \quad \partial S / \partial \phi_{2}=\text { const }
$$

we obtain solutions for the odd Grassmann variables:

$$
\psi=\psi_{0} \exp \left(-i \int U(q(\tau)) \mathrm{d} \tau\right), \quad \bar{\psi}=\bar{\psi}_{0} \exp \left(i \int U(q(\tau)) \mathrm{d} \tau\right)
$$

Let us introduce the following series for the even Grassmann variable:

$$
q(t)=x_{q c}(t)+q_{0}(t) \bar{\psi} \psi=x_{q c}(t)+q_{0}(t) \bar{\psi}_{0} \psi_{0}
$$

Then, from the Jacobi theorem we have:

$$
-\frac{\partial S}{\partial A}=\int \frac{\mathrm{d} q}{\sqrt{2 E-V^{2}(q)}} \bar{\psi}_{0} \psi_{0}-t \bar{\psi}_{0} \psi_{0}=\text { const. }
$$

From (18) and (19) we can write

$$
\int \frac{\mathrm{d} x_{q c}}{\sqrt{2 E-V^{2}\left(x_{q c}\right)}}-t=\text { const. }
$$

Let us now evaluate the derivative $\partial S / \partial E=$ const. Taking into account the result $(20)$ and the following expansions

$$
\begin{aligned}
& U(q)=U\left(x_{q c}\right)+U^{\prime}\left(x_{q c}\right) q_{0} \bar{\psi}_{0} \psi_{0}, \\
& V^{2}(q)=V^{2}\left(x_{q c}\right)+2 V^{\prime}\left(x_{q c}\right) V\left(x_{q c}\right) q_{0} \bar{\psi}_{0} \psi_{0}, \\
& f\left(V^{2}(q)\right)=f\left(V^{2}\left(x_{q c}\right)\right)+f^{\prime}\left(V^{2}\left(x_{q c}\right)\right) 2 V^{\prime}\left(x_{q c}\right) V\left(x_{q c}\right) q_{0} \bar{\psi}_{0} \psi_{0},
\end{aligned}
$$

we obtain: 


$$
\int \frac{\mathrm{d} q_{0}}{\sqrt{2 E-V^{2}\left(x_{q c}\right)}}=\int \frac{\left[A-U\left(x_{q c}(\tau)\right)-V\left(x_{q c}(\tau)\right) V^{\prime}\left(x_{q c}(\tau)\right) q_{0}(\tau)\right]}{2 E-V^{2}\left(x_{q c}(\tau)\right)} \mathrm{d} \tau
$$

This result can be presented in the form:

$$
q_{0}(t)=\frac{\dot{x}_{q c}(t)}{\dot{x}_{q c}(0)}\left[q_{0}(0)-\int_{0}^{t} \mathrm{~d} \tau \frac{F-U\left(x_{q c}(\tau)\right)}{2 E-V^{2}\left(x_{q c}(\tau)\right)}\right] .
$$

The obtained result coincides with the result obtained from the Lagrangian equations of motion [1].

Thus the Hamilton-Jacobi equation and Jacobi theorem are presented in Grassmann algebra. The action for the classical system characterized by the SUSY Lagrangian is presented in the explicit form. The results obtained using the Hamilton-Jacobi method coincide with the ones obtained previously from the Lagrangian equations of motion.

\section{ACKNOWLEDGEMENTS}

I am very grateful to Dr. V. M. Tkachuk for comments and discussions.
[1] A. Inomata, G. Junker, Phys. Rev. A 50, 3638 (1994).

[2] G. Junker, M. Stephan, J. Phys A 28, 1467 (1995).

[3] A. Inomata, G. Junker, S. Matthiesen, preprint hep-th 9510230 (1995).

[4] E. Witten, Nucl. Phys. B 188, 513 (1981).

[5] R. Hojman, J. Zanelli, Phys. Rev. D 35, 3825 (1987).

[6] P. A. M. Dirac, Lectures on Quantum Mechanics. (Belfer
Graduate school of Science, Yeshiva Univ., New York 1964).

[7] E. S. Fradkin, I. V. Tyutin, Phys. Rev. D 2, 2841 (1970).

[8] D. M. Gitman, I. V. Tyutin, Kanonicheskoie kvantovaniie poliei so sviaziami (Quantization of fields with constraints) (Nauka Press, Moscow, 1986).

[9] V. P. Akulov, S. Duplij, preprint hep-th 9809089 (1998).

\title{
МЕТОД ГАМІЛЬТОНА-ЯКОБІ ДЛЯ КЛАСИЧНОЇ МЕХАНІКИ, ПОБУДОВАНОЇ В АЛГЕБРІ ГРАСМАНА
}

\author{
K. В. Табуншик \\ Інститут фізики конденсованих систем НАН Украйни, \\ вул. Свенціцького, 1, Львіб, 79011, Украӥна
}

\begin{abstract}
У прапі рівняння Гамільтона-Якобі й теорема Якобі записані для класичної механіки в алгебрі Грасмана. Для псевдокласичної системи, яка в частковому випадку є суперсиметричною, знайдено загальний інтеграл рівняння Гамільтона-Якобі. За допомогою теореми Якобі отримано загальний інтеграл рівнянь руху, що збігається з розв'язком рівнянь Лагранжа-Ейлера.
\end{abstract}

